\title{
From Static Code Distribution to More Shrinkage for the Multiterminal Cut
}

\author{
Bram De Wachter ${ }^{\star}$, Alexandre Genon ${ }^{\star}$, and Thierry Massart \\ Université Libre de Bruxelles \\ Département d'Informatique, Bld du Triomphe, B-1050 Bruxelles \\ \{bdewacht, agenon, tmassart\}@ulb.ac.be
}

\begin{abstract}
We present the problem of statically distributing instructions of a common programming language, a problem which we prove equivalent to the multiterminal cut problem. We design efficient shrinkage techniques which allow to reduce the size of an instance in such a way that optimal solutions are preserved. We design and evaluate a fast local heuristics that yields remarkably good results compared to a well known $2-\frac{2}{k}$ approximation algorithm. The use of the shrinkage criterion allows us to increase the size of the instances solved exactly, or to augments the precision of any particular heuristics.
\end{abstract}

\section{Introduction}

We present the problem of automatic distribution of a programming language, motivated by our research in automatic distributed industrial control systems [16]. This problem consists in distributing a program code among different sites, minimizing the total communications between these sites during its execution. We show that this problem is NP-hard. Furthermore, we show that it is equivalent to the multiterminal cut presented in [6], and therefore concentrate on finding new ways to attack the problem described in terms of multiterminal cut.

The key concept used in this paper is based on shrinkage, a notion presented by Dahlhaus et al. in [6] where an instance $I$ is transformed into a smaller instance $I^{\prime}$ in such a way that all optimal solutions in $I^{\prime}$ can be easily transformed into optimal solutions for $I$.

In this paper, we generalize the shrinkage criterion of Dahlhaus et al. which is based on st cuts, to all nodes in the instance graph and prove its correctness. Then, we present an implementation of a fast local heuristics taking advantage of this new shrinkage operation. The heuristics combines both the new shrinkage based reduction and an unshackle operation which operates on graphs where no more shrinkage is possible.

We also introduce maximum size minimum st cuts, and prove (theorems 5, 6 and 7) some unexpected properties on the structure of these cuts. We exploit these results to obtain a more efficient implementation for our shrinkage algorithm, and prove (theorem 8) that the procedure of Goldberg and Tarjan, presented in [9] for max-flow/min-cut actually computes these cuts. A practical evaluation is presented showing that our heuristics yields generally better results

* Work supported by the Region de Bruxelles Capitale, grant no. RBC-BR 227/3298. 
than the approximation algorithm designed by Dahlhaus et al. To the best of our knowledge, we perform the first experimental study of the approximation algorithm of [6].

\section{Optimal static code distribution is hard}

Our problem consists in finding, at compile time, an optimal distribution of an imperative regular program. Such a program contains instructions (assignments, loops and tests), a set of static global internal variables and a set of static global I/O variables. The distributed environment in which the program runs is composed of several sites, each of which contains some of the global I/O variables. A correct distribution is an assignment of all variables and all instructions to the set of sites such that the following distribution constraints are satisfied : (1) the $\mathrm{I} / \mathrm{O}$ variables are on the predefined sites, (2) each variable and instruction is on exactly one site, (3) each instruction using a variable is on the site of that variable.

The assignment of instructions to sites influences the performance of the program during execution: each time control flows from an instruction assigned to one site to an instruction assigned to another site, the executed distribution environment must synchronize (e.g. by sending a message over a network) in order to continue the execution on the other site. The optimal distribution is such that the expected number of (synchronization) messages exchanged during execution is minimum. In order to evaluate this performance criterion, we suppose that a realistic control flow frequency function $\mathcal{W}$ is given, expressing the expected number of times control flows from one instruction to another.

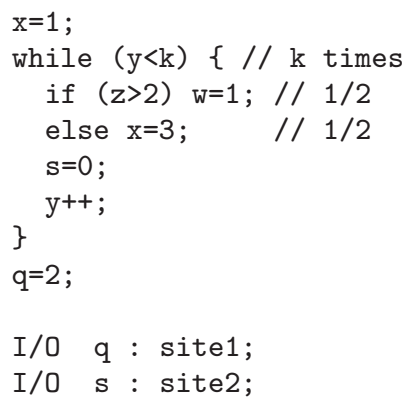

(a)

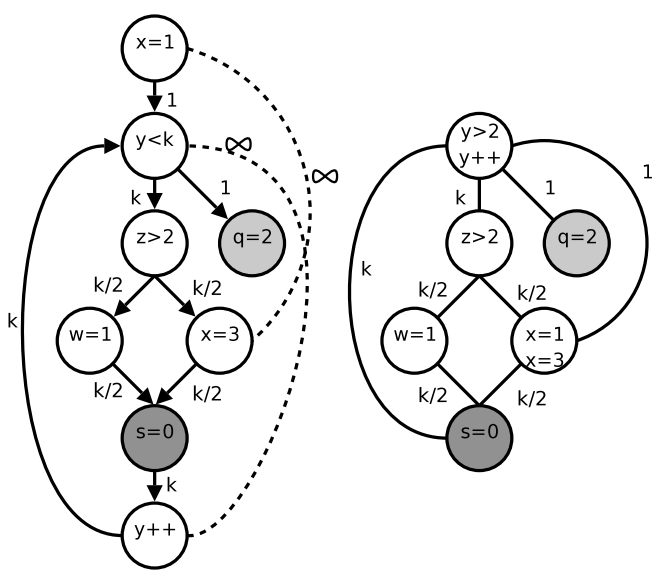

(b) (c)

Fig. 1. The distribution problem

A graphical presentation of the optimal distribution problem can be found in figure 1. The program of figure 1(a) can be graphically modeled by its control 
flow graph (figure 1(b)) where the nodes are its instructions and edges model the control flow between instructions with weights defined by $\mathcal{W}$. The graph of figure 1(c) is the undirected graph where all nodes using the same variables are merged. We say that this graph is the result of the merging of $x=1$ and $x=3$ and of the merging of $\mathrm{y}>\mathrm{k}$ and $\mathrm{y}++$ in the first graph. More formally, when two nodes $n$ and $n^{\prime}$ are merged, $n$ and $n^{\prime}$ are replaced by one new node $n^{\prime \prime}$, and all edges $\{v, n\}$ and $\left\{u, n^{\prime}\right\}$ are changed to $\left\{v, n^{\prime \prime}\right\}$ and $\left\{u, n^{\prime \prime}\right\}$. Note that when more than one edge exists between two nodes, all of the edges between those nodes can be replaced by a single edge weighted by the sum of the weights of these edges. Remark that both representations of figure 1 are equivalent with respect to the optimal distribution problem. The merging operation is sometimes called contraction if an edge exists between two merged nodes, since that edge would disappear from the graph. We now give a formal definition of the multiterminal cut problem on weighted undirected graphs.

Definition 1 (Multiterminal cut problem). Given a weighted undirected graph $G(V, E, w): E \subseteq\{\{u, v\} \mid u, v \in V \wedge u \neq v\}^{1}, w: E \mapsto \mathbb{N}$ and a set of terminals $T=\left\{s_{1}, \ldots, s_{k}\right\} \subseteq V$, find a partition of $V$ into $V_{1}, \ldots, V_{k}$ such that $s_{i} \in V_{i} \forall i \in[1, k]$ and $\sum_{v \in V_{i}, v^{\prime} \in V_{j}, i \neq j} w\left(v, v^{\prime}\right)$ is minimized.

We know that the multiterminal cut problem is NP-Hard [6] for fixed $k>2$, even when all weights are equal to 1 . As shown in appendix A, optimal distribution is an NP-hard problem. The following theorems, proved in appendix A, state that the optimal distribution problem and the multiterminal cut are equivalent.

Theorem 1. There exists a polynomial time reduction from the optimal distribution problem to the multiterminal cut.

Theorem 2. There exists a polynomial time reduction from multiterminal cut on unweighted graphs to the optimal distribution problem.

With these two theorems, we can conclude that the optimal distribution problem is polynomially equivalent to the multiterminal cut. Thus, to solve the optimal distribution problem, we can concentrate on the multiterminal cut in the program's control flow graph.

\section{Related Works}

The multiterminal cut problem has first been studied by Dahlhaus et al. in [6]. In this paper, the authors prove that this problem is NP-hard for $k>2$ even when $k$ is fixed where $k$ is the number of terminals. The problem is polynomially solvable when $k=2$, a well known result proved by Ford and Fulkerson [7], and in the case of planar graphs. The authors also present a $2-\frac{2}{k}$ polynomial time approximation algorithm that relies on isolating cuts, a technique that is detailed further on. Moreover, they proved that this problem is MAX SNP-hard, i.e. there is no polynomial time approximation scheme unless $\mathrm{P}=\mathrm{NP}$. In [1], Calinescu, Karloff,

\footnotetext{
${ }^{1}$ For technical reasons looping edges $(v, v)$ will be omitted in all graphs considered here. Note that their presence does not change the problem.
} 
and Rabani, presented a linear programming relaxation. Using this technique and a well chosen rounding procedure, they obtain an approximation factor of $1.5-\frac{1}{k}$. This factor was lowered to 1.3438 by Karger et al. in [12] who give better approximations when $k \geq 14$. These improvements were found by studying carefully the integrality gap and giving a more precise rounding procedure. A polyhedral approach $[2,14,4]$ and a non-linear formulation [5] have also been studied for the multiterminal cut problem.

Shrinkage has also been studied by Högstedt and Kimelman in [10]. In this paper, the authors give some optimality-preserving heuristics that allow to reduce the size of the input graph by contracting some edges. The shrinkage technique presented here generalizes some of their criteria (such as independent nets and articulation points).

In this paper, we consider the multiterminal cut problem on undirected graphs, but work has also been done on directed graphs. Naor and Zosin presented a 2-approximation algorithm for this problem in [13]. On the other hand, Costa, Letocart and Roupin proved in [3] that multiterminal cuts on acyclic graphs could be computed in polynomial time using a simple flow algorithm. A generalization of multiterminal cut is minimum multicut where a list of pairs of terminals is given and we must find a set of edges such that these pairs of terminals are disconnected. Garg et al. [8] give a $O(\log k)$-approximation algorithm for this minimum multicut. A survey on multiterminal cuts and its variations can be found in [3].

The applications that rely on the multiterminal cut fall mainly into two domains : the domain of parallel computation and the partitioning of distributed applications. The problems encountered in parallel computation are concerned with the allocation of tasks on different processors. The total load must be partitioned in roughly equal sized pieces, characterized by some load balancing criterion, and this subject to some interconnection criterion that must be minimized ([11] and [15]). These problems can be formulated using the strongly related $k$-cut problem, which asks to partition the graph in $k$ subsets such that crossing edges are minimized. Since this problem has no fixed terminals, it is polynomially solvable, for any fixed $k \geq 3[6]$ and is thus considerably easier than the problem addressed here.

For the distributed applications, the problem is similar, only that it is the several application's components that must be distributed among different processors. Several criteria are studied, such as the inter object communication load of [10]. However, we are not aware of other works that are based on the static distribution of the instructions where the control flow is used to minimize the expected communications load. Because of this fine grain distribution, the scale of our problem is considerably larger than the studies on the partitioning of objects or functions as is the case in classical distributed systems. Therefore, we believe that the results of the heuristics presented here are applicable on these smaller instances as well.

\section{A generalized global criterion}

In [6] the authors design a $2-\frac{2}{k}$ approximation algorithm based on the isolation heuristics which uses st cuts. An st cut (multiterminal cut with $k=2$ ) divides 
the graph into two sets $(C, \bar{C})$ where $s \in C$ and $t \in \bar{C}$. The heuristics consists in finding an optimal isolating cut for each of the $k$ terminals $\left\{s_{1}, \ldots, s_{k}\right\}$ and taking the union of the $k-1$ smallest of these cuts. An optimal isolating cut is a minimum st cut where $\mathrm{s}=s_{i}$ and $\mathrm{t}$ is the node resulting of the merging of $s_{j \neq i}$. We now introduce the original shrinkage theorem proved by Dahlhaus et al :

Theorem 3 (Shrinkage). Given graph $G(V, E, w)$ with terminals $T=\left\{s_{1}\right.$, $\left.\ldots, s_{k}\right\} \subseteq V$. Let $G_{i}^{\prime}$ be the graph where all terminals in $T \backslash\left\{s_{i}\right\}$ are merged into $t$, and $(C, \bar{C})$ the st cut between $s_{i}$ and $t$, then there exists an optimal multiterminal cut $\left(V_{1}, \ldots, V_{k}\right)$ of $G$ such that $\exists \ell: C \subseteq V_{\ell}$.

Theorem 3 allows us to shrink (i.e. to merge) all nodes in $C$ into one node. Shrinkage is clearly an interesting way to attack the multiterminal cut problem. Indeed, we can apply theorem 3 to all terminal nodes in order to shrink the graph. And if one can obtain a relatively small instance, then there may be hope to find the optimal solution by exhaustive search. It can also be used independently of any other algorithm designed to approximate the multiterminal cut problem. We extend theorem 3 to handle more shrinkage as follows :

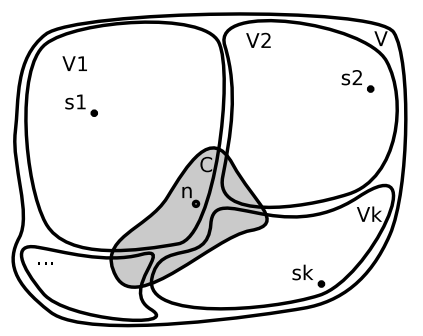

Fig. 2. Theorem 4

Theorem 4 (More shrinkage). Given graph $G(V, E, w)$ with terminals $T=$ $\left\{s_{1}, \ldots, s_{k}\right\} \subseteq V$. Let $v \in V$, and $G_{v}^{\prime}$ be the graph where all terminals in $T \backslash\{v\}$ are merged into $t$, and $(C, \bar{C})$ the minimum st cut between $v$ and $t$ in $G_{v}^{\prime}$ then there exists an optimal multiterminal cut of $G\left(V_{1}, \ldots, V_{k}\right)$ such that $\exists \ell: C \subseteq V_{\ell}$.

Proof. Outline (a detailed proof can be found in appendix B). Figure 2 illustrates the proof for $l=1$. Proof by contradiction. Take any minimum multiterminal cut $C^{*}=\left(V_{1}, V_{2}, \ldots, V_{k}\right)$ and suppose that $v \in V_{1}$ but $C \nsubseteq V_{1}$. Take $C^{*^{\prime}}=$ $\left(V_{1} \cup C, V_{2} \backslash C, \ldots, V_{k} \backslash C\right)$. Then we can show that the weight of $C^{*^{\prime}} \leq$ the weight of $C^{*}$ by using the weights of the edges between $C$ and $\bar{C}$ and between $C \cap V_{1}$ and $\overline{C \cap V_{1}}$ : since $(C, \bar{C})$ is a minimum st cut, the border of $V_{1}$ can be extended to $V_{1} \cup C$ without increasing the multiterminal cut's weight.

Theorem 3 differs from this theorem because we can apply the former only on terminal nodes, while the latter can be applied to all nodes in the graph, resulting in more shrinkage and therefore smaller graphs.

We now explain how to use theorem 4 to shrink an instance of the multiterminal cut problem. Let $s \in V$, we compute the st cut were $t$ is the result 
of the merging of all terminals in $T \backslash s$. The nodes that are in the same partition as $s$ are merged together, with theorem 4 assuring that this preserves opimality. A chain of graphs $G_{1}, \ldots, G_{l}$ can therefore be calculated where each graph is the result of the optimal merging with respect to its predecessor, and where $G_{l}$ can not be reduced any further. To compute these st cuts, one can use the algorithm of Goldberg and Tarjan [9], with complexity $O\left(n m \log \frac{n^{2}}{m}\right)$ (where $n=|V|, m=|E|)$. With the results contained in the next section, we can show that when this well known algorithm is used, then $l \leq n$, resulting in a total complexity in $O\left(n^{2} m \log \frac{n^{2}}{m}\right)$.

Once a graph cannot be reduced any further, two options remain, either search exhaustively and find an optimal solution, or unshackle the graph. Unshackling means contracting one or more edges that likely connect nodes from the same partition in the optimal cut. Note that if an edge is picked that is in every optimal multiterminal cut, this operation will not preserve optimality. Once the graph is unshackled, the resulting graph may be ready for further optimal reductions. In the following section, we study an implementation using the shrinkage technique combined with a fast local unshackling heuristics.

\section{A fast local heuristics}

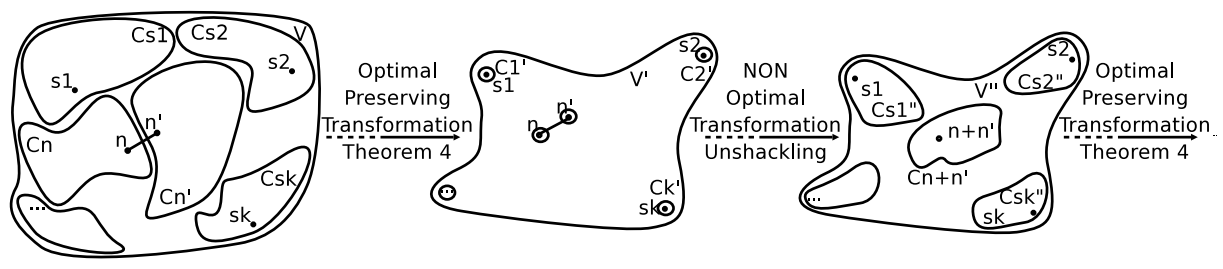

Fig. 3. Optimal and non optimal reductions

As said in previous section, we can use the shrinkage technique in combination with an unshackling heuristics. Figure 3 gives a graphical overview of this technique and figure 4 presents an implementation. We first perform shrinkage until the graph cannot be reduced any further. Then, we use an unshackling heuristics to contract one edge from this graph. The shrinkage technique may thereupon be reused on this unshackled graph. This process is repeated until the graph contains only terminal nodes. While the resulting multiterminal cut may not be optimal, due to the unshackling heuristics, we will see that this technique generally computes a fairly good multiterminal cut and is quite efficient, provided that the unshackling is easy to compute.

\subsection{Definition and complexity}

Definition 2 (MAX-MIN-st cut). Given graph $G(V, E, w)$ and two different nodes $s, t \in V$. Define min-ST $(s, t)$ as the set of cuts separating $s$ and $t$ with 


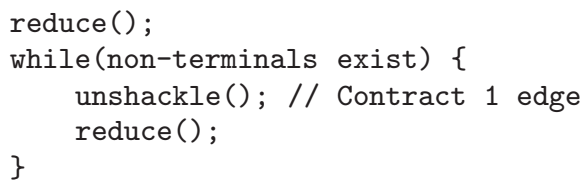

Fig. 4. Unshackling heuristics

minimum weight. We define the set MAX-MIN-st $(s, t)$ as the set of nodes left connected to $s$ by the cut $(C, \bar{C}) \in \min -S T(s, t)$ such that $|C|$ is maximal. We can easily extend these definitions for sets of nodes. For a set $T$, MAX-MIN-st $(s, T)$ is equivalent to MAX-MIN-st $(s, t)$ in the graph $G$ where all nodes in $T$ have been merged into the new node $t$.

We now prove some interesting properties related to maximum size minimum cuts. Theorems 5, 6 and 7 give some remarkable insights on the structure of these cuts, which leads to a more efficient implementation of our heuristics. We use the following notation :

$w(A, B)=\sum_{\{(x, y) \mid x \in A, y \in B\}} w(x, y)$, and let $w(X)=w(X, \bar{X})$, where $\bar{X}=V \backslash X$.

Theorem 5. Given graph $G(V, E, w)$ and two nodes $s, t \in V$, MAX-MiN-st $(s, t)$ is uniquely defined, i.e. there is only one maximal size minimum st cut for any couple $(s, t)$.

Proof. Outline (a full proof can be found in appendix B). By contradiction : suppose $S$ and $S^{\prime}\left(S \neq S^{\prime}\right)$ both satisfy the definition of MAX-MIN-st $(s, t)$. Let $I=S \cap S^{\prime}$ and $T=V \backslash\left(S \cup S^{\prime}\right)$, it is easy to see that $S$ (resp. $\left.S^{\prime}\right) \neq I$, else $S$ (resp. $S^{\prime}$ ) would not be a maximal size minimum st cut. First, since $S$ is a min st cut we start from $w(I) \geq w(S)$ to prove that $w(S \backslash I, I) \geq w(S \backslash I, T)$. Next, we compute $w\left(S \cup S^{\prime}\right)$, note that $S \cup S^{\prime}$ is also an st cut for $(s, t)$. We show that $w\left(S \cup S^{\prime}\right)=w(S \backslash I, T)+w\left(S^{\prime}, T\right) \leq w\left(S^{\prime}\right)$. Finally, observe that $S \cup S^{\prime}$ is therefore a minimum st cut for $(s, t)$ with larger cardinality than $S$ or $S^{\prime}$.

Theorem 6. For any three nodes $s, s^{\prime}, t$ of $V$, if $s^{\prime} \in \operatorname{MAX}-\operatorname{Min}-\mathrm{st}(s, t)$, then MAX-MiN-st $\left(s^{\prime}, t\right) \subseteq$ MAX-MIN-st $(s, t)$.

Proof. By contradiction : let $S=$ MAX-MiN-st $(s, t), S^{\prime}=\operatorname{MAX}-\min -\mathrm{st}\left(s^{\prime}, t\right)$ and suppose that $S^{\prime} \nsubseteq S$. We have that $\left|S \cup S^{\prime}\right|>|S|$. Let $I=S \cap S^{\prime}$ and $T=$ $V \backslash\left(S \cup S^{\prime}\right)$, we define the following :

$$
\begin{array}{rlrl}
A \equiv w(S \backslash I, T) & B \equiv w(I, T) & C \equiv w\left(S^{\prime} \backslash I, T\right) \\
D \equiv w(I, S \backslash I) & E \equiv w\left(I, S^{\prime} \backslash I\right) & F \equiv w\left(S \backslash I, S^{\prime} \backslash I\right) \\
\Longrightarrow & w\left(S \cup S^{\prime}\right)=A+B+C & w(S)=A+B+E+F \\
& w\left(S^{\prime}\right)=B+C+D+F & w(I)=B+D+E
\end{array}
$$

From the definition of $S$ and $S^{\prime}$ we have $w\left(S \cup S^{\prime}\right)>w(S)$ (as $S \subsetneq S \cup S^{\prime}$ ) and $w(I) \geq w\left(S^{\prime}\right)$, which implies that $C>E+F$ and $E \geq C+F$. This leads to a contradiction.

Theorem 7. Given graph $G(V, E, w)$ and three distinct nodes $s, s^{\prime}, t \in V$. Let $S=\operatorname{MAX}-\mathrm{MiN-st}(s, t), S^{\prime}=\operatorname{MAX}-\min -\operatorname{st}\left(s^{\prime}, t\right), I=S \cap S^{\prime}$, and $T=V \backslash$ 
$\left(S \cup S^{\prime}\right)$. If $I \neq \emptyset$ and $S \neq I$ and $S^{\prime} \neq I$, then $w(I, S \backslash I)=w\left(I, S^{\prime} \backslash I\right)$. Moreover, we have that $w\left(I, V \backslash\left(S \cup S^{\prime}\right)\right)=0$. The same results hold when $S=$ MAX-Min-st $\left(s,\left\{t \cup s^{\prime}\right\}\right), S^{\prime}=$ MAX-MIN-st $\left(s^{\prime}, t\right)$ or $S=\operatorname{MAX}$-MIN-st $\left(s,\left\{t \cup s^{\prime}\right\}\right)$, $S^{\prime}=$ MAX-MIN-st $\left(s^{\prime},\{s \cup t\}\right)$.

Proof. By contradiction : let's reuse the equations from proof of theorem 6, to compute $w(S \backslash I)$ and $w(S)$ :

$$
w(S \backslash I)=A+D+F \quad w(S)=A+B+E+F
$$

As $S$ is the MAX-Min-st cut $(s, t)$, we have $A+B+E+F \leq A+D+F$ and $B+E \leq D$. By applying a similar reasoning with $S^{\prime}$ and $S^{\prime} \backslash I$, we can prove that $B+D \leq E$. In conclusion, we have $E=D\left(\Rightarrow w(I, S \backslash I)=w\left(I, S^{\prime} \backslash I\right)\right)$ and $B(\equiv w(I, T))=0$. The two other propositions are proved likewise.

Theorems 5, 6 and 7 allow us to efficiently calculate the reduction phases of our unshackling heuristics. We know that the order in which we calculate the cuts has no effect on the outcome of the algorithm. Moreover, we can calculate the MAX-MIN-st cut for a given node $n$ and immediately merge all nodes on the same side of $n$ in the cut, thus reducing the number of nodes before calculating the next MAX-MIN-st cut for the remaining unmodified nodes. After the calculation and merging of all MAX-MIN-st cuts, we have for all nodes in the reduced graph and terminals $s_{1}, \ldots, s_{k}$, MAX-MIN-st cut $\left(s, \cup_{i} s_{i} \backslash\{s\}\right)=\{s\}$. The only missing link is how to calculate MAX-MIN-st:

Theorem 8. The algorithm of Goldberg and Tarjan [9] calculating the maximum flow in $O\left(n m \log \left(\frac{n^{2}}{m}\right)\right)$-time also calculates MAX-MIN-st

Proof. By contradiction. As for prerequisites, the reader is expected to be familiar with [9], where the authors prove that it is possible to calculate a minimum st cut $\left(S_{g}, \bar{S}_{g}\right)$ with $s \in S_{g} \wedge t \in \bar{S}_{g}$ in $O\left(n m \log \left(\frac{n^{2}}{m}\right)\right)$-time. We will use their notations to prove that the min st cut calculated by their algorithm is in fact the unique minimum st cut of maximal size.

Let $g(v, w): E \mapsto \mathbb{R}^{+}$be the preflow function (here we may suppose that the algorithm terminated and that the preflow is a legal flow). $G_{g}$ is used to indicate the residual graph and $c(v, w): E \mapsto \mathbb{R}^{+}$indicates the capacities of the edges in $E$. In addition, $\left(S_{g}, \bar{S}_{g}\right)$ is defined as the partition of $V$ such that $\bar{S}_{g}$ contains all nodes from which $t$ is reachable in $G_{g}$ and $S_{g}=V \backslash \bar{S}_{g}$.

We use the following lemma by Golberg and Tarjan from [9]:

When the first stage terminates, $\left(S_{g}, \bar{S}_{g}\right)$ is a cut such that every pair $v, w$ with $v \in S_{g}$ and $w \in \bar{S}_{g}$ satisfies $g(v, w)=c(v, w)$.

Suppose that there exists another minimum cut $\left(C^{\prime}, \overline{C^{\prime}}\right)$ such that $\left|C^{\prime}\right|>\left|S_{g}\right|$ which is maximal in size.

Remark that $S_{g} \subseteq C^{\prime}$ because of theorem 6 and $s \in C^{\prime} \cap S_{g}$.

Let $I=C^{\prime} \cap \bar{S}_{g}$. Note that $I \neq \emptyset$ since $\left|C^{\prime}\right|>\left|S_{g}\right|$. We split the boundaries between $S_{g}, I$ and $\bar{S}_{g}$ in three sets :

- Old Boundary: $O \subseteq E=(v, w): v \in S_{g} \backslash I \wedge w \in I$ 
- New Boundary: $N \subseteq E=(v, w): v \in I \wedge w \in \bar{S}_{g} \backslash I$

- Common Boundary: $C \subseteq E=(v, w): v \in S_{g} \backslash I \wedge w \in \bar{S}_{g} \backslash I$

By definition of $\left(S_{g}, \bar{S}_{g}\right)$, we know that $g(O)=c(O)$. We also know that since $\left(C^{\prime}, \overline{C^{\prime}}\right)$ and $\left(S_{g}, \bar{S}_{g}\right)$ are both minimum cuts : $w(O)+w(C)=w(N)+w(C) \Rightarrow$ $w(O)=w(N)$. Remark that since $g$ is a legal flow, the flow entering $I$ must be equal to the flow getting out of $I$, which means that $g(O)=g(N)$.

The combination of these tree equations leads to a contradiction: since the edges in $N$ are saturated, $t$ is not reachable from any $n \in I$ in $G_{g}$ which means that $I=\emptyset$.

Finally, we can prove that the worst execution time for the unshackling heuristics stays within the complexity of the reduction algorithm :

Theorem 9. The unshackling algorithm from figure 4 can be implemented with worst case complexity $O\left(n^{2} m \log \left(\frac{n^{2}}{m}\right)\right)$ if the complexity of unshackle() is in $O\left(n m \log \left(\frac{n^{2}}{m}\right)\right)$.

Proof. Consider an irreducible graph in which one and only one edge $\left\{v_{1}, v_{2}\right\}$ is contracted. Before contraction, $\forall v \in V:$ MAX-MIN-st $(v, T)=\{v\}$. It is easy to see (proof by contradiction) that after contraction $\forall v \in V \backslash\left\{v_{1}, v_{2}\right\}$ : MAX-MIN-st $(v, T)=\{v\}$, i.e. the contraction only affects the resulting node from the contraction, which means that after each contraction only one MAX-MIN-st cut has to be calculated. Since at most $n$ contractions are possible, the number of MAX-MIN-st calculations needed can be bounded by $2 n$ ( $n$ for the initial reduction and $n$ for all subsequent reductions). The worst case complexity is therefore as stated, if the complexity of unshackle() is $O\left(n m \log \left(\frac{n^{2}}{m}\right)\right)$.

\subsection{Results}

It remains to define the way we will unshackle the graph. We tried several local procedures, among which :

- Greedy : take an edge with maximal weight.

- Error-reduction : take an edge where the expected error is small

- Balanced weight : take an edge $\left\{n_{1}, n_{2}\right\}$ such that $\sum_{\left\{\left\{n_{1}, n^{\prime}\right\} \in E\right\}} w\left(n_{1}, n^{\prime}\right)+\sum_{\left\{\left\{n_{2}, n^{\prime}\right\} \in E\right\}} w\left(n_{2}, n^{\prime}\right)$ is maximal

- Max-unshackle : take an edge that has high reduction rate

Surprisingly, we found that balanced weight works much better than the others. Unfortunately, we discovered that none of these heuristics have a fixed approximation bound, however, since our calculations include the ones from the $k-\frac{2}{k}$ approximation algorithm, we can compare the results and take the better of both, resulting in the same bound without any extra cost. In order to compare both heuristics, this has not been done in the following experiments.

Two sets of experiments were conducted : figures $5(\mathrm{a}), 5(\mathrm{~b})$ show the results on random graphs, while $5(\mathrm{c})$ shows the results on graphs obtained from auto generated programs. 


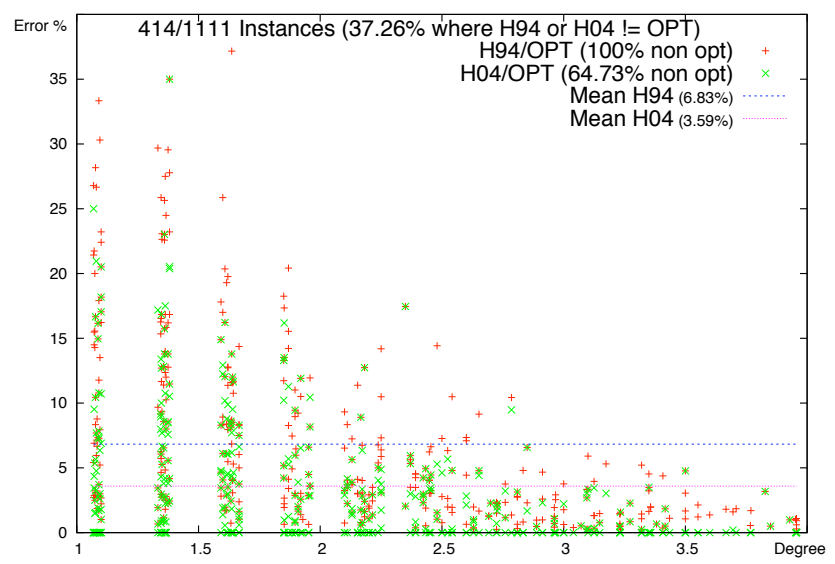

(a) Unshackling heuristics on random graphs

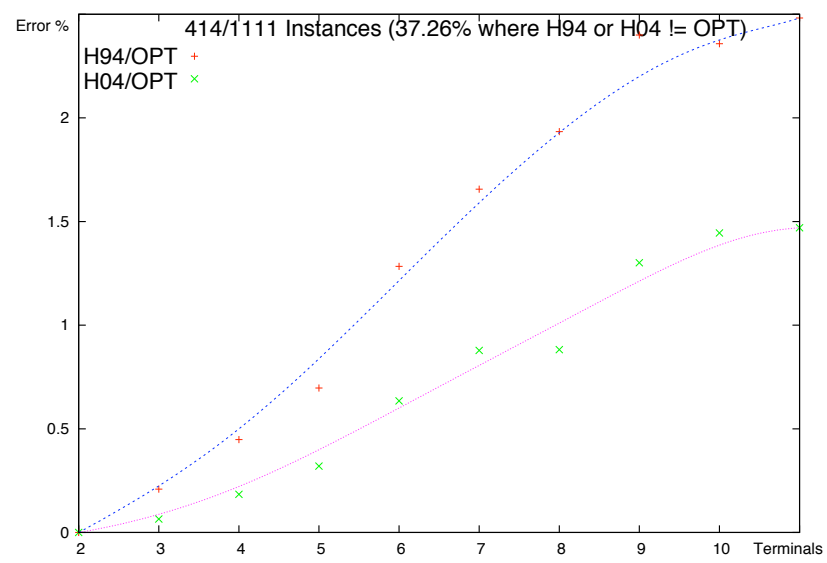

(b) Unshackling heuristics on random graphs

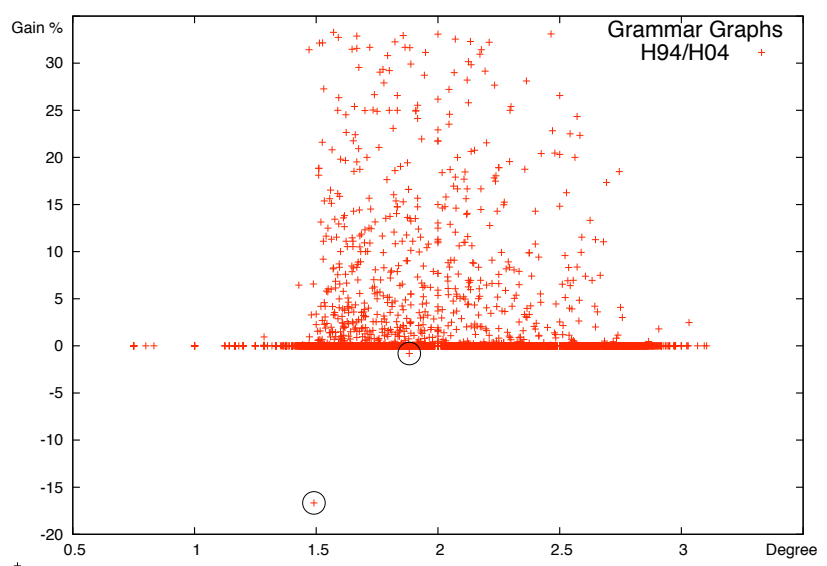

(c) Unshackling heuristics on grammar graphs

Fig. 5. Results for the unshackling heuristics 
In figure $5(\mathrm{a})$ we compare the results of our heuristics (indicated by $\mathcal{H} 04$ ) with the approximation algorithm (called $\mathcal{H} 94$ ) from Dahlhaus et al. 1111 experiments where conducted on sufficiently small graphs (ranging from 20-40 nodes), allowing us to compare with the optimal solution. For 411 hard cases (37\%), one of the heuristics failed to find the optimal. We can see that for increasing mean degree (X-axis), the error rate (Y-axis, in percent w.r.t. the optimal) for both algorithms drops rapidly, caused by the randomness in the graph. For sparse graphs however, error rates can be as high as $35 \%$. The mean error rate for $\mathcal{H} 04$, for these hard cases, is $3.6 \%$ while it raises to $6.8 \%$ for $\mathcal{H} 94$. Remark that the failure rate for $\mathcal{H} 94$ is $100 \%$ of the hard cases, while our algorithm failed in $65 \%$ of these cases. In these experiments, there was no instance where $\mathrm{H}_{4} 4$ performed worse compared to $\mathcal{H} 94$.

Figure 5(b) shows the mean error rate (Y-axis, in \% w.r.t. the optimal solution) for the experiments of figure 5(a), with increasing number of terminals (X-axis). We can clearly see the gain of our algorithm.

Figure 5(c) shows results for 25.000 grammar graphs of moderate size (600 nodes, 3 to 10 terminals), where the two algorithms are compared to each other. $\mathrm{X}$-axis gives the mean degree. We can observe a difference of as high as $+35 \%$ (Y-axis) for some cases, meaning that our algorithm improves the other by the same amount. For only 2 instances, our algorithm performed worse (1.3\% worse and $14 \%$ worse).

\section{Conclusions}

In this paper, we studied the problem of optimal code distribution of an imperative regular program, a problem equivalent to the multiterminal cut problem.

We presented a criterion that allows to perform shrinkage on a given graph such that optimal solutions for the multiterminal cut problem on the resulting graph are optimal solutions for the original graph. This criterion is a generalization of the criterion of Dahlhaus et al. presented in [6]. Using this shrinkage criterion, we designed a heuristics that, in practice, finds near optimal solutions for the multiterminal cut problem. In our search for an efficient implementation for our shrinkage algorithm, we defined maximum size minimum cuts for wich we prove some interesting structural properties.

Future works We hope that structural properties can lead to a more thorough understanding of the combinatorial structure of optimal solutions for the multiterminal cut. Furthermore, we are currently searching other unshackling procedures that could be combined with our shrinkage technique. Moreover, we are trying to determine why the balanced weight unshackling performs well in practice.

We are also considering the use of our shrinkage technique in the branch and bound context. We are currently integrating our shrinkage technique into the CPLEX solver in order to speed up the general branch and bound phase of the mixed integer optimizer. 


\section{References}

1. Gruia Calinescu, Howard Karloff, and Yuval Rabani. An improved approximation algorithm for multiway cut. J. Comput. Syst. Sci., 60(3):564-574, 2000.

2. Sunil Chopra and Jonathan H. Owen. Extended formulations for the a-cut problem. Math. Program., 73:7-30, 1996.

3. M. Costa, L. Letocart, and F. Roupin. Minimal multicut and maximal integer multiflow: a survey. European Journal of Operational Research, 162(1):55-69, 2005.

4. W.H. Cunningham. The optimal multiterminal cut problem. DIMACS series in discrete mathematics and theoretical computer science, 5:105-120, 1991.

5. R. Vohra D. Bertsimas, C. Teo. Nonlinear formulations and improved randomized approximation algorithms for multicut problems. In Proc. 4th conference on integer programming and combinatorial optimization, volume 920 of $L N C S$, pages 29-39, 1995.

6. Elias Dahlhaus, David S. Johnson, Christos H. Papadimitriou, P. D. Seymour, and Mihalis Yannakakis. The complexity of multiterminal cuts. SIAM J. Comput., 23(4):864-894, 1994.

7. L.R. Ford and D.R. Fulkerson. Flows in Networks. Princeton University Press, Princeton, NJ, 1962.

8. Naveen Garg, Vijay V. Vazirani, and Mihalis Yannakakis. Multiway cuts in directed and node weighted graphs. In Proceedings of the 21st International Colloquium on Automata, Languages and Programming, pages 487498. Springer-Verlag, 1994.

9. Andrew V. Goldberg and Robert E. Tarjan. A new approach to the maximum-flow problem. Journal of the ACM (JACM), 35(4):921-940, October 1988. ISSN:0004-5411.

10. K. Hogstedt and D. Kimelman. Graph cutting algorithms for distributed applications partitioning. SIGMETRICS Performance Evaluation Review, 28(4):27-29, 2001.

11. Lisa Hollermann, Tsan sheng Hsu, Dian Rae Lopez, and Keith Vertanen. Scheduling problems in a practical allocation model. J. Comb. Optim., 1(2):129-149, 1997.

12. David R. Karger, Philip Klein, Cliff Stein, Mikkel Thorup, and Neal E. Young. Rounding algorithms for a geometric embedding of minimum multiway cut. In STOC '99: Proceedings of the thirty-first annual ACM symposium on Theory of computing, pages 668-678, 1999.

13. J. Naor and L. Zosin. A 2-approximation algorithm for the directed multiway cut problem. SIAM J. Comput., 31(2):477-482, 2001.

14. M.R. Rao S. Chopra. On the multiway cut polyhedron. Networks, 21:51-89, 1991.

15. Tsan sheng Hsu, Joseph C. Lee, Dian Rae Lopez, and William A. Royce. Task allocation on a network of processors. IEEE Trans. Computers, 49(12):1339-1353, 2000.

16. Bram De Wachter, Thierry Massart, and Cédric Meuter. dsl : An environment with automatic code distribution for industrial control systems. In Principles of Distributed Systems, 7th International Conference, OPODIS 2003, volume 3144 of $L N C S$, pages 132-145. Springer, 2004. 


\section{A From optimal distribution to multiterminal cut}

In order to show that both problems are equivalent, we need to formally define the optimal distribution problem. Let $\mathcal{W}$ be a realistic control flow frequency function. Although calculating such a function is undecidable in general, profiling tools and monitoring can result in good approximations. Note that a realistic control flow frequency function $\mathcal{W}$ for common languages will be such that $\mathcal{W}\left(i, i^{\prime}\right)=\mathcal{W}\left(i^{\prime}, i^{\prime \prime}\right)$ if $i, i^{\prime}$ and $i^{\prime \prime}$ are sequential instructions, $\mathcal{W}\left(i, i^{\prime}\right)=k \mathcal{W}\left(i^{\prime \prime}, i^{\prime \prime \prime}\right)$ if $i, i^{\prime}$ is in a loop that is executed $k$ times relative to the instructions $i^{\prime \prime}, i^{\prime \prime \prime}$ outside the loop, and $\mathcal{W}\left(i, i^{\prime}\right)=\frac{1}{p} \mathcal{W}\left(i^{\prime \prime}, i^{\prime \prime \prime}\right), p \in[0,1]$ if $i, i^{\prime}$ is in a conditional IF branch with respect to instructions $i^{\prime \prime}, i^{\prime \prime \prime}$ outside the IF. We suppose that $p \in \mathbb{Q}$ so that every weight can be multiplied by a common factor to obtain an equivalent instance of the problem with natural weights.

Definition 3 (The optimal distribution problem). Given a certain program $P$ (variables $V$, instructions $I$, flow estimation $\mathcal{W}$ ), a set of $I / O$ variables $\mathcal{V} \subseteq V$, a set of sites $S$, and an assignment of the $I / O$ variables to the sites $\mathcal{C}: \mathcal{V} \mapsto S$, find a total assignment $C: V \mapsto S$, respecting the distribution constraints, such that $\sum_{\{i, j \in I \mid C(i) \neq C(j)\}} \mathcal{W}(i, j)$ is minimum.

We now recall the definition of multiterminal cut :

Definition 4 (Multiterminal cut problem). Given a weighted undirected graph $G(V, E, w): E \subseteq\{\{u, v\} \mid u, v \in V \wedge u \neq v\}^{2}, w: E \mapsto \mathbb{N}$ and a set of terminals $T=\left\{s_{1}, \ldots, s_{k}\right\} \subseteq V$, find a partition of $V$ into $V_{1}, \ldots, V_{k}$ such that $s_{i} \in V_{i} \forall i \in[1, k]$ and $\sum_{v \in V_{i}, v^{\prime} \in V_{j}, i \neq j} w\left(v, v^{\prime}\right)$ is minimized.

We know that the multiterminal cut problem is NP-Hard [6] for fixed $k>2$, even when all weights are equal to 1 . We now show that both problems are equivalent.

Theorem 10. There exists a polynomial-time reduction from the optimal distribution problem to the multiterminal cut.

Proof. Take the control flow graph $G$ of the optimal distribution problem weighted by $\mathcal{W}$, and merge all instructions using the same variables into one node. This way, we only have at most one node using a given variable. Let $G^{\prime}$ be this new graph, and let $T=\left\{s_{1}, s_{2}, \ldots, s_{k}\right\}$ be the set of nodes of $G^{\prime}$ using a I/O variable. It is easy to see that $G^{\prime}, T$ is an instance of the multiterminal cut and that an optimal solution to this instance is an optimal distribution of the original program. Indeed, starting from a multiterminal cut $V_{1}, \ldots, V_{k}$, we can build an optimal partition $P_{1}, \ldots, P_{k}$ such that $P_{i}$ is the set of instructions such that their nodes in the control flow graph is in $V_{i}$. Note that $G^{\prime}, T$ can be computed from $G$ in polynomial time.

The first theorem states that we can easily solve the optimal distribution problem using multiterminal cut. From this theorem, we can deduce the following corollary :

\footnotetext{
${ }^{2}$ For technical reasons looping edges $(v, v)$ will be omitted in all graphs considered here. Note that their presence does not change the problem.
} 
Corollary 1. Let $P$ be a program, and $G_{f}$ its control flow graph after merging, it is equivalent to solve the optimal distribution on $P$ or to solve the multiterminal cut on $G_{f}$.

Proof. If we have an optimal distribution $P_{1}, \ldots, P_{k}$ of $P$, then we can easily compute a multiterminal cut $V_{1}, \ldots, V_{k}$ for $G_{f}$ by taking the same partition. The constraint that every node of $G_{f}$ is in only one $S_{i}$ is respected because of the constraints on correct distributions. Moreover, it is easy to see that $V_{1}, \ldots, V_{k}$ is optimal an multiterminal cut. The other direction is a direct consequence of theorem 10.

Next lemma states that, starting from an unweighted graph, we can build a program such that the control flow graph, after merging, is equal to the original graph (except for a constant factor 2 between the weights on the edges).

Lemma 1. Let $G(V, E)$ be an unweighted graph, then there exists a program $P$ such that the control flow graph $G_{f}=\left(V_{f}, E_{f}, w_{f}\right)$ of $P$, after merging, is such that $V=V_{f}, E=E_{f}$, and $\forall\left\{v, v^{\prime}\right\} \in E, w_{f}\left(\left\{v, v^{\prime}\right\}\right)=2$.

Proof. Let $G(V, E)$, we can build a program $P$ such that, after having merged nodes using the same variables in the control flow graph, the resulting graph $G_{f}$ is equal to $G$. Moreover, this program contains only a list of assignments. We define the set of global variables as $X_{V}=\left\{x_{v} \mid v \in V\right\}$, i.e. there is a bijective mapping between the set $V$ and the set of global variables $X_{V}$. We show the construction of $P$ by induction on the size of $V$. The case where $V=\{v\}$ (i.e. $|V|=1)$ is obvious, we only have one instruction $i \equiv x_{v} \leftarrow 1$.

Suppose therefore that $|V|=n$, and let $P$ be the corresponding program using instructions $\left\{x_{u} \leftarrow 1 \mid u \in V\right\}$. We now build a program $P^{\prime}$ for the graph $G^{\prime}=$ $\left(V^{\prime}=V \cup\{v\}, E^{\prime}=E \cup\left\{\left\{v, v_{1}\right\}, \ldots\left\{v, v_{\ell}\right\}\right\}\right.$. For this, we modify $P$ as follows : For all $j \in[1, \ell]$ (i.e. for all new edges), let $x_{v_{j}}$ be the global variable corresponding to $v_{j}$, and let $i \equiv x_{v_{j}} \leftarrow 1$ be an instruction of $P$. In $P^{\prime}$, we replace $i$ by : $i ; x_{v} \leftarrow 1 ; i$. Note that $P^{\prime}$ is still a list of assignments. Let $G_{f}^{\prime}$ be the control flow graph of $P^{\prime}$, after merging, we have that $G_{f}^{\prime}$ contains all edges of $E^{\prime}$. And, if we set $\mathcal{W}$ such that it assigns 1 between two consecutive instructions (which is the case for common programming languages), then all edge weights in $G_{f}^{\prime}$ will be equal to 2 .

Note that the number of assignments of $P$ is polynomial in the size of $G$. Since all edges in $G_{f}$ have weights equal to 2 , it is easy to see that $G$ and $G_{f}$ have the same optimal solution for the multiterminal cut.

Theorem 11. There exists a polynomial time reduction from multiterminal cut on unweighted graphs to the optimal distribution problem.

Proof. Let $G(V, E), T$ be an unweighted instance of multiterminal cut, by lemma 1, we can build a program $P$ which has its control flow graph $G_{f}$ after merging equal to $G$ (except for the constant factor of 2 on the weights). From corollary 1, we know that it is equivalent to solve the multiterminal cut on $G_{f}$ than to solve the optimal distribution on $P$. 
We know that multiterminal cut on unweighted graphs is NP-hard. Thus, we can state the following corollary.

Corollary 2. The optimal distribution problem is NP-hard, even when the program contains only a sequence of instructions.

Corollary 3. The optimal distribution problem is polynomially equivalent to the multiterminal cut problem on arbitrary graphs.

This results from theorems 10 and 11 . Arbitrary weights $2 \omega$ can be obtained in the construction of theorem 11 using the while construct as follows : replace the sequence $i ; x_{v} \leftarrow 1 ; i$ with $i ; x_{v} \leftarrow w$; while $\left(x_{v}>1\right)\left\{i ; x_{v} \leftarrow x_{v}-1\right\} i$. 


\section{B Proofs}

Theorem 4 (More shrinkage). Given graph $G(V, E, w)$ with terminals $T=$ $\left\{s_{1}, \ldots, s_{k}\right\} \subseteq V$. Let $v \in V$, and $G_{v}^{\prime}$ be the graph where all terminals in $T \backslash\{v\}$ are merged into $t$, and $(C, \bar{C})$ the minimum st cut between $v$ and $t$ in $G_{v}^{\prime}$ then there exists an optimal multiterminal cut of $G\left(V_{1}, \ldots, V_{k}\right)$ such that $\exists \ell: C \subseteq V_{\ell}$.

Proof. Suppose a minimum multiterminal cut $C^{*}$ and, without any loss of generality, $l=1\left(\right.$ i.e. $\left.v \in V_{1}\right)$. We define a partition $C^{*^{\prime}}$ of $V$ in $V_{1}^{\prime}, \ldots, V_{k}^{\prime}$ as follows :

$$
\begin{aligned}
V_{1}^{\prime} & =V_{1} \cup C \\
V_{j \neq 1}^{\prime} & =V_{j} \backslash\left(V_{j} \cap C\right)=V_{j} \backslash C
\end{aligned}
$$

We show that $C^{*^{\prime}}$ is a multiterminal cut with weight less than (or equal to) the weight of $C^{*}$, proving the existence of a multiterminal cut described in the theorem. It is easy to verify that $C^{*^{\prime}}$ is a multiterminal cut, since

$-V_{i}^{\prime} \cap V_{j \neq i}^{\prime}=\emptyset$

$-\cup_{i} V_{i}^{\prime}=V$

$-\forall i: s_{i} \in V_{i}^{\prime}$

- $\forall i: s_{i}$ is isolated from $s_{j \neq i}$

Let us show that $C^{*^{\prime}}$ has weight less than (or equal to) the weight of $C^{*}$. Let $A, B \subseteq V, A \cap B=\emptyset$, we note $w(A, B)=\sum_{\{(x, y) \mid x \in A, y \in B\}} w(x, y)$. Furthermore, let $w(X)=w(X, \bar{X})$, where $\bar{X}=V \backslash X$. Using these notations, we have the following rules :

$$
\begin{array}{rlr}
w(X, Y) & =w(Y, X) & \\
w(X, Y \cup Z) & =w(X, Y)+w(X, Z)-w(x, Y \cap Z) & \\
w(X, Y \cup Z) & =w(X, Y)+w(X, Z) & \\
w(X, Y \backslash Z) & =w(X, Y)-w(X, Y \cap Z) & \text { if } Y \cap Z=\emptyset
\end{array}
$$

The weights of the two multiterminal cuts $C^{*^{\prime}}$ and $C^{*}$ can be expressed in terms of their partitions (resp. $V_{i}^{\prime}$ and $V_{i}$ ) as follows :

$$
\begin{aligned}
w\left(C^{*^{\prime}}\right) & =\sum_{j \neq 1} w\left(V_{1}^{\prime}, V_{j}^{\prime}\right)+\sum_{i \neq 1, j>i} w\left(V_{i}^{\prime}, V_{j}^{\prime}\right) \\
w\left(C^{*}\right) & =\sum_{j \neq 1} w\left(V_{1}, V_{j}\right)+\sum_{i \neq 1, j>i} w\left(V_{i}, V_{j}\right)
\end{aligned}
$$


In addition, we use two st cuts for proving $w\left(C^{*^{\prime}}\right) \leq w\left(C^{*}\right)$ :

$$
\begin{array}{rlr}
w(C) & =w(C, V \backslash C)=w\left(C,\left(\cup_{j} V_{j}\right) \backslash C\right) & \\
& =\sum_{j} w\left(C, V_{j} \backslash C\right) & \\
& =w\left(C, V_{1} \backslash C\right)+\sum_{j \neq 1} w\left(C, V_{j} \backslash C\right) & \\
w\left(C \cap V_{1}\right) & =w\left(C \cap V_{1}, V \backslash\left(C \cap V_{1}\right)\right) & \\
& =w\left(C \cap V_{1},\left(\cup_{j} V_{j}\right) \backslash\left(C \cap V_{1}\right)\right. & \\
& =\sum_{j} w\left(C \cap V_{1}, V_{j} \backslash\left(C \cap V_{1}\right)\right) & \text { by (4), (5) } \\
& =w\left(C \cap V_{1}, V_{1} \backslash C\right)+\sum_{j \neq 1} w\left(C \cap V_{1}, V_{j}\right) &
\end{array}
$$

In order to calculate $w\left(C^{*^{\prime}}\right)-w\left(C^{*}\right)$, we will express all terms of $C^{*^{\prime}}$ in terms of $C^{*}$ :

$$
\begin{aligned}
w\left(V_{1}^{\prime}, V_{j \neq 1}^{\prime}\right)= & w\left(V_{1} \cup C, V_{j} \backslash C\right) & & \text { by (1), (2) } \\
= & w\left(V_{j} \backslash C, V_{1}\right)+w\left(V_{j} \backslash C, C\right) & & \text { by (3), (4) } \\
& -w\left(V_{j} \backslash C, V_{1} \cap C\right) & & \\
= & w\left(V_{1}, V_{j}\right)-w\left(V_{1}, V_{j} \cap C\right)+w\left(V_{j} \backslash C, C\right) & & \text { by (3), (6) } \\
& -w\left(V_{1} \cap C, V_{j}\right)+w\left(V_{1} \cap C, V_{j} \cap C\right) & & \text { (2) } \\
w\left(V_{i \neq 1}^{\prime}, V_{j>i}^{\prime}\right)= & w\left(V_{i} \backslash C, V_{j} \backslash C\right) & & \text { by } \\
= & w\left(V_{i} \backslash C, V_{j}\right)-w\left(V_{i} \backslash C, V_{j} \cap C\right) & & \text { by (6) } \\
= & w\left(V_{j}, V_{i}\right)-w\left(V_{j}, V_{i} \cap C\right) & & \text { by (3), (6) } \\
& -w\left(V_{j} \cap C, V_{i}\right)+w\left(V_{j} \cap C, V_{i} \cap C\right) & &
\end{aligned}
$$

Using equations (14) and (15) in (7), we can express the difference between $C^{*^{\prime}}$ and $C^{*}$ using (10) and (13) :

$w\left(C^{*^{\prime}}\right)-w\left(C^{*}\right)=$

$$
\begin{aligned}
& w(C)-w\left(C \cap V_{1}\right) \\
& +\sum_{j \neq 1}\left(-w\left(V_{1}, V_{j} \cap C\right)+w\left(V_{1} \cap C, V_{j} \cap C\right)\right) \\
& +\sum_{i \neq 1, j>i}\left(-w\left(V_{j}, V_{i} \cap C\right)-w\left(V_{j} \cap C, V_{i}\right)+w\left(V_{i} \cap C, V_{j} \cap C\right)\right) \\
& -w\left(V_{1} \backslash C, C\right)+w\left(V_{1} \cap C, V_{1} \backslash C\right)
\end{aligned}
$$

which proves the theorem since

- (16) $\leq 0$ because $C$ is a minimum cut (remark that $C \cap V_{1}$ is a st cut between $v$ and $t)$ 
- (17) $\leq 0$ because $\left(V_{1} \cap C\right) \subseteq V_{1}$

- (18) $\leq 0$ because $\left(V_{i} \cap C\right) \subseteq V_{i}$ and $w(X, Y) \geq 0$

- (19) $\leq 0$ because $\left(V_{1} \cap C\right) \subseteq C$

Theorem 5. Given graph $G(V, E, w)$ and two nodes $s, t \in V$, MAX-MiN-st $(s, t)$ is uniquely defined, i.e. there is only one maximal size minimum st cut for any couple $(s, t)$

Proof. By contradiction. Suppose $S$ and $S^{\prime}\left(S \neq S^{\prime}\right)$ both satisfy the definition of MAX-Min-st $(s, t)$ Let $I=S \cap S^{\prime}$ and $T=V \backslash\left(S \cup S^{\prime}\right)$, it is easy to see that $S$ (resp. $\left.S^{\prime}\right) \neq I$, else $S$ (resp. $S^{\prime}$ ) would not be a maximal size minimum st cut. As $S$ is a min st cut, we have :

$$
\begin{aligned}
w(I) & \geq w(S) \\
\Longleftrightarrow w(S \backslash I, I)+w\left(S^{\prime} \backslash I, I\right)+w(I, T) & \geq w\left(S, S^{\prime} \backslash I\right)+w(S, T) \\
\Longleftrightarrow w(S \backslash I, I)+w\left(S^{\prime} \backslash I, I\right)+w(I, T) \geq & w\left(S, S^{\prime} \backslash I\right)+w(S \backslash I, T) \\
& +w(I, T) \\
\Longleftrightarrow w(S \backslash I, I)+w\left(S^{\prime} \backslash I, I\right) \geq & w\left(S, S^{\prime} \backslash I\right)+w(S \backslash I, T)
\end{aligned}
$$

As $I \subseteq S$, we have that

$$
w\left(S^{\prime} \backslash I, I\right) \quad\left(=w\left(I, S^{\prime} \backslash I\right)\right) \leq w\left(S, S^{\prime} \backslash I\right)
$$

Therefore, we must have

$$
w(S \backslash I, I) \geq w(S \backslash I, T)
$$

Now, let's compute $w\left(S \cup S^{\prime}\right)$, note that $S \cup S^{\prime}$ is also a st cut for $(s, t)$.

$$
\begin{array}{rlrl}
w\left(S \cup S^{\prime}\right) & =w(S \backslash I, T)+w\left(S^{\prime} \backslash I, T\right)+w(I, T) & & \\
& \leq w(S \backslash I, I)+\underbrace{w\left(S^{\prime} \backslash I, T\right)+w(I, T)}_{=w\left(S^{\prime}, T\right)} & & \text { by } 20 \\
& \leq w(S \backslash I, I)+w\left(S^{\prime}, T\right) & \\
& \leq w\left(S \backslash I, S^{\prime}\right)+w\left(S^{\prime}, T\right) & & \text { since } I \subseteq S^{\prime} \\
& \leq w\left(S^{\prime}\right) & &
\end{array}
$$

Thus, $S \cup S^{\prime}$ is a minimum st cut for $(s, t)$. But we have $S^{\prime} \subsetneq S \cup S^{\prime}$, therefore $S, S^{\prime} \notin$ MAX-MiN-st $(s, t)$ and we have a contradiction. 